\title{
Channel Coding for Interference Alignment Onto a Lattice for Real-Valued Channels
}

\author{
Cibele C. Trinca \\ UNICAMP - Departamento de Comunicações \\ Campus Campinas \\ 13083-872, Campinas, SP \\ E-mail: cibtrinca@yahoo.com.br, \\ Jean-Claude Belfiore \\ Télécom ParisTech - Department of Communications and Electronics \\ Campus Paris, Sophia Antipolis \\ F-75634 Cedex 13, Paris, FRANCE \\ E-mail: belfiore@enst.fr,

\section{Edson D. de Carvalho} \\ UNESP - Departamento de Matemática \\ Campus Ilha Solteira \\ 15385-000, Ilha Solteira, SP \\ E-mail: edson@mat.feis.unesp.br,

\section{Jozué V. Filho} \\ UNESP - Curso de Engenharia de Telecomunicações \\ Campus São João da Boa Vista \\ 13870-090, São João da Boa Vista, SP \\ E-mail: jozue.vieira@sjbv.unesp.br.
}

\begin{abstract}
Interference is usually viewed as an obstacle to communication in wireless networks, so we developed a new methodology to quantize the channel coefficients in order to realize interference alignment onto a lattice. Our channel model is the same as the compute-and-forward strategy. In this work, we suppose that our interference channel is real-valued and we describe a way to find a nested lattice partition chain, for any dimension $n=2^{r-2}$, where $r \geq 3$, in order to quantize the channel coefficients. For that, we make use of the maximal real subfield $K$ of $L=\mathbb{Q}\left(\xi_{2^{r}}\right)$, where $r \geq 3, \xi=\xi_{2^{r}}$ is the $2^{r}$-th root of unity and $K=\mathbb{Q}(\theta)$, with $\theta=\xi+\xi^{-1}$.
\end{abstract}

Palavras-chave: Channel Coding, Lattice, Inteference Alignment

\section{Introduction}

By [2], we know that the compute-and-forward strategy enables relays to decode linear equations of the transmitted messages by using the noisy linear combinations provided by the channel and we have an equivalent channel induced by the modulo- $\Lambda$ transformation. In this "virtual" channel model, each relay observes a $\mathbb{Z}[i]$-combination $\sum a_{m l} t_{l}$ of the lattice points 
corrupted by effective noise $z_{e q, m}$, that is,

$$
y_{m}=\sum_{l=1}^{L} a_{m l} t_{l}+z_{e q, m} .
$$

In this work, we suppose that our interference channel is real-valued, specifically $a_{m l} \in \mathbb{R}$, and, in order to realize interference alignment onto a lattice, we need to quantize the channel coefficients $a_{m l}$. We describe a way to find a nested lattice partition chain, for any dimension $n=2^{r-2}$, where $r \geq 3$, in order to quantize the channel coefficients. For that, we make use of the maximal real subfield $K$ of $L=\mathbb{Q}\left(\xi_{2^{r}}\right)$, where $r \geq 3, \xi=\xi_{2^{r}}$ is the $2^{r}$-th root of unity and $K=\mathbb{Q}(\theta)$, with $\theta=\xi+\xi^{-1}$.

We also have that the coding scheme only requires that each relay knows the channel coefficients from each transmitter to itself. Specifically, relay $m$ only needs to know $a_{m l}$. Each transmitter only needs to know the desired message rate, not the realization of the channel.

For this new methodology, we introduce an error criterion that measures, in a probabilistic sense, the error between the desired quantity and our estimate of it. Therefore, we focus on choosing our estimate to minimize the expected or mean value of the square of the error, referred to as a minimum mean-square-error (MMSE) criterion.

But, in this work, we only discuss about the methodology related to the channel approximation in order to realize interference alignment onto a lattice.

\section{Quantization of the Channel Gains}

As described in the previous section, suppose that our interference channel is real-valued, specifically $a_{m l} \in \mathbb{R}$. We suppose that all lattices used by the legitimate user and the interferers are one of a certain lattice partition chain and extended by periodicity. Now the idea we want to develop is that the effect of a channel gain on a given user is to shift the lattice used by the user either to the left, if its channel gain is smaller than 1 , or to the right, if it is larger than 1 . It is very important that the channel gain does not remove the lattice from the initial chain of nested lattices.

In this section, we consider $n$-dimensional real-valued vectors, where $n=2^{r-2}$ and $r \geq 3$. Now we write, for a given user, how its codeword can be transformed so that we can perform the channel quantization. We make use of the maximal real subfield $K$ of $L=\mathbb{Q}\left(\xi_{2^{r}}\right)$, where $r \geq 3, \xi=\xi_{2^{r}}$ is the $2^{r}$-th root of unity and $K=\mathbb{Q}(\theta)$, with $\theta=\xi+\xi^{-1}$.

So we consider the following Galois extensions, where $r \geq 3$ :

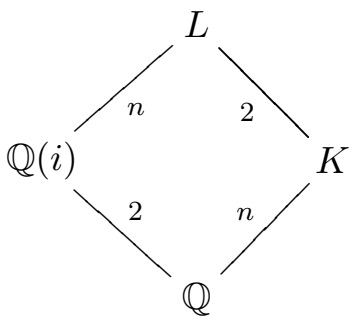

Let $\xi=\xi_{2^{r}}$ be a $2^{r}$-th root of unity, where $r \geq 3$. Let $L=\mathbb{Q}(\xi)=K(i)$ and $K=$ $\mathbb{Q}(\theta)$ be the maximal real subfield of $L$, where $\theta=\xi+\xi^{-1}$. By the theorem 10, in [1], we have that $[L: \mathbb{Q}]=2^{r-1},[K: \mathbb{Q}]=2^{r-2}=n, O_{K}=\mathbb{Z}[\theta]$ is the ring of integers of $K$ and $\left\{1, \xi+\xi^{-1}, \ldots, \xi^{n-1}+\xi^{-(n-1)}\right\}$ is an integral basis of $O_{K}$.

As $\left[\mathbb{Q}\left(\xi_{2^{r}}\right): \mathbb{Q}\right]=\phi\left(2^{r}\right)=2^{r-1}$, where $\phi$ is the Euler function, and $[\mathbb{Q}(i): \mathbb{Q}]=2$, then we have $\left[\mathbb{Q}\left(\xi_{2^{r}}\right): \mathbb{Q}(i)\right]=2^{r-2}=n$.

Let $\operatorname{Gal}(K / \mathbb{Q})=\left\{\sigma_{1}, \sigma_{2}, \ldots, \sigma_{n}\right\}$ be the Galois group of $K$ over $\mathbb{Q}([1]$, pg. 6). 
Let's find an ideal of norm equal to 2, that is, we find an element of $O_{K}$ with absolute algebraic norm equal to 2 . In fact,

$$
\begin{gathered}
2=N_{L / \mathbb{Q}}(1+\xi)=N_{K / \mathbb{Q}}\left(N_{L / K}(1+\xi)\right)= \\
=N_{K / \mathbb{Q}}\left(1+\xi^{-1}+\xi+1\right)=N_{K / \mathbb{Q}}\left(2+2 \cos \left(\pi / 2^{(r-1)}\right)\right) .
\end{gathered}
$$

Observe that $\xi+\xi^{-1}=\theta=2 \cos \left(\pi / 2^{(r-1)}\right)$, then $N_{K / \mathbb{Q}}(2+\theta)=2$. So

$$
N_{K / \mathbb{Q}}(p)=2 \text {, where } p=(2+\theta) \in O_{K} .
$$

Therefore $\Im=\langle p\rangle=\langle 2+\theta\rangle=p O_{K}$ is a principal ideal of norm 2. By [1], pg. 3, we have that

$$
R^{T}=\left(\begin{array}{ccc}
\sqrt{\sigma_{1}(\alpha)} \sigma_{1}\left(\alpha_{1}\right) & \cdots & \sqrt{\sigma_{1}(\alpha)} \sigma_{1}\left(\alpha_{n}\right) \\
\sqrt{\sigma_{2}(\alpha)} \sigma_{2}\left(\alpha_{1}\right) & \cdots & \sqrt{\sigma_{2}(\alpha)} \sigma_{2}\left(\alpha_{n}\right) \\
\vdots & \ddots & \vdots \\
\sqrt{\sigma_{n}(\alpha)} \sigma_{n}\left(\alpha_{1}\right) & \cdots & \sqrt{\sigma_{n}(\alpha)} \sigma_{n}\left(\alpha_{n}\right)
\end{array}\right)
$$

is a generator matrix of the lattice $\Lambda=\left\{x=M \lambda \mid \lambda \in \mathbb{Z}^{n}\right\}$, where $\alpha \in K$ is totally positive, that is, $\sigma_{i}(\alpha)>0$, for all $i=1,2, \ldots, n$, and $\left\{\alpha_{1}, \alpha_{2}, \ldots, \alpha_{n}\right\}$ is a basis of $A$ over $\mathbb{Z}$, where $A \subseteq O_{K}$ is an ideal.

In this case, we have that the Gram matrix $\left(R^{T}\right)^{T} R^{T}=R R^{T}$ coincides with the trace form $\left(\operatorname{Tr}\left(\alpha \alpha_{i} \alpha_{j}\right)\right)_{i, j=1}^{n}$, where $T$ denotes the transposition.

We know that $\left\{1, \xi+\xi^{-1}, \ldots, \xi^{n-1}+\xi^{-(n-1)}\right\}=\left\{e_{0}, e_{1}, \ldots, e_{(n-1)}\right\}$ is an integral basis of $O_{K}$. Observe in [1], pg. 6 , that $T$ is a basis transformation matrix from $\left\{e_{i}\right\}_{i=0}^{n-1}$ to $\left\{f_{i}\right\}_{i=0}^{n-1}$, where $f_{i}=-\sum_{j=0}^{i} e_{j}$, for all $i=0,1,2, \ldots, n-1$, and $T=T^{T}$. So $\left\{f_{0}, f_{1}, \ldots, f_{n-1}\right\}$ is another basis ( $\mathbb{Z}$-basis) of $O_{K}$.

Finally, the generator matrix of the rotated $\mathbb{Z}^{n}$-lattice is given by $([1]$, pg. 7$)$

$$
R=\frac{1}{\sqrt{2^{r-1}}} T M A .
$$

But, in this work, the columns of a matrix generate the $\mathbb{Z}^{n}$-lattice, then $M_{0}=R^{T}$ generates the rotated $\mathbb{Z}^{n}$-lattice $\Lambda_{0}$. So $\Lambda_{0}=\left\{x=M_{0} \lambda \mid \lambda \in \mathbb{Z}^{n}\right\} \simeq \mathbb{Z}^{n}$ and $M_{0}=\frac{1}{\sqrt{2^{r-1}}} A M^{T} T=$ $\frac{1}{\sqrt{2^{r-1}}} A M^{\prime}\left(M^{\prime}=M^{T} T\right)$, where

$$
\begin{gathered}
M=\left(\begin{array}{ccc}
\sigma_{1}\left(e_{0}\right) & \cdots & \sigma_{n}\left(e_{0}\right) \\
\vdots & \ddots & \vdots \\
\sigma_{1}\left(e_{n-1}\right) & \cdots & \sigma_{n}\left(e_{n-1}\right)
\end{array}\right), \\
A=\operatorname{diag}\left(\sqrt{\sigma_{k}(\alpha)}\right)_{k=1}^{n}, \text { where } \alpha=2-\theta, \text { and } \\
T=\left(\begin{array}{ccccc}
-1 & -1 & -1 & \cdots & -1 \\
-1 & -1 & -1 & \cdots & 0 \\
\vdots & \vdots & \vdots & \ddots & \vdots \\
-1 & 0 & 0 & \cdots & 0
\end{array}\right) .
\end{gathered}
$$

But $M^{\prime}=M^{T} T$ can be directly calculated if we use the new basis $\left\{f_{i}\right\}_{i=0}^{n-1}$, that is,

$$
M^{\prime}=\left(\begin{array}{ccc}
\sigma_{1}\left(f_{0}\right) & \cdots & \sigma_{1}\left(f_{n-1}\right) \\
\sigma_{2}\left(f_{0}\right) & \cdots & \sigma_{2}\left(f_{n-1}\right) \\
\vdots & \ddots & \vdots \\
\sigma_{n}\left(f_{0}\right) & \cdots & \sigma_{n}\left(f_{n-1}\right)
\end{array}\right) .
$$


Observe, by [1], that $R R^{T}=I$, where $I$ is the identity matrix. Then

$$
M_{0}^{T} M_{0}=\left(R^{T}\right)^{T} R^{T}=R R^{T}=I .
$$

At the receiver, we suppose that we apply $M_{0}$ to the received vector of (1) to get

$$
\bar{y}_{m}=M_{0} y_{m}=\sum_{l=1}^{L} a_{m l} M_{0} t_{l}+M_{0} z_{e q, m} .
$$

As $z_{e q, m}$ is i.i.d. circularly symmetric complex Gaussian noise and $M_{0}$ is orthogonal, then the noise in (9) is also i.i.d. circularly symmetric complex Gaussian. Now let's take a look at the vectors of the form $a_{m l} M_{0} t_{l}$. For sake of simplicity of notations, we denote each one of these vectors by

$$
\bar{x}=h \cdot M_{0} \cdot x,
$$

where $x=t_{l}$ is the lattice point transmitted by the considered user and $h=a_{m l}$ is the channel coefficient. We can rewrite it now as

$$
\left(\begin{array}{cccc}
h & 0 & \cdots & 0 \\
0 & h & \cdots & 0 \\
\vdots & \vdots & \ddots & \vdots \\
0 & 0 & \cdots & h
\end{array}\right) \cdot U \cdot x=H \cdot U \cdot x .
$$

We can observe that $\Im^{k}, k \in \mathbb{Z}$, is an ideal of $O_{K}$ generated by $p^{k}$, with $p=2+\theta$. So $\Im^{k}=p^{k} O_{K}$.

Now, if $\left\{\alpha_{1}, \alpha_{2}, \ldots, \alpha_{n}\right\}$ is a $\mathbb{Z}$-basis of $O_{K}$, then we can see that

$$
\left\{p^{k} \alpha_{1}, p^{k} \alpha_{2}, \ldots, p^{k} \alpha_{n}\right\}
$$

is a $\mathbb{Z}$-basis of $\Im^{k}=\left\langle p^{k}\right\rangle=p^{k} O_{K}$, since the set of invertible fractional ideals form an abelian group related to the product of ideals.

We know, by [1], that $\left\{f_{0}, f_{1}, \ldots, f_{n-1}\right\}$ is a $\mathbb{Z}$-basis of $O_{K}$ used to find the rotated $\mathbb{Z}^{n}$-lattice $\Lambda_{0}$. So, by previously, it follows that $\left\{p^{k} f_{0}, p^{k} f_{1}, \ldots, p^{k} f_{n-1}\right\}$ is a $\mathbb{Z}$-basis of $\Im^{k}=p^{k} O_{K}$. Then the generator matrix $M_{k}$ of the lattice $\Lambda_{k}=\left\{x=\left(M_{k}\right) \lambda \mid \lambda \in \mathbb{Z}^{n}\right\}$ is given by

$$
\begin{aligned}
& M_{k}=\left(\begin{array}{ccc}
\sqrt{\sigma_{1}(\alpha)} \sigma_{1}\left(p^{k} f_{0}\right) & \cdots & \sqrt{\sigma_{1}(\alpha)} \sigma_{1}\left(p^{k} f_{n-1}\right) \\
\sqrt{\sigma_{2}(\alpha)} \sigma_{2}\left(p^{k} f_{0}\right) & \cdots & \sqrt{\sigma_{2}(\alpha)} \sigma_{2}\left(p^{k} f_{n-1}\right) \\
\vdots & \ddots & \vdots \\
\sqrt{\sigma_{n}(\alpha)} \sigma_{n}\left(p^{k} f_{0}\right) & \cdots & \sqrt{\sigma_{n}(\alpha)} \sigma_{n}\left(p^{k} f_{n-1}\right)
\end{array}\right)= \\
& =A \cdot\left(\begin{array}{cccc}
\sigma_{1}\left(p^{k}\right) & 0 & \cdots & 0 \\
0 & \sigma_{2}\left(p^{k}\right) & \cdots & 0 \\
\vdots & \vdots & \ddots & \vdots \\
0 & 0 & \cdots & \sigma_{n}\left(p^{k}\right)
\end{array}\right) \cdot M^{\prime}=A A^{\prime} M^{T} T
\end{aligned}
$$

where $A^{\prime}=\operatorname{diag}\left(\sigma_{i}\left(p^{k}\right)\right)_{i=1}^{n}$.

As $A$ and $A^{\prime}$ are diagonal matrices, we have $A A^{\prime}=A^{\prime} A$. Then $M_{k}=A^{\prime}\left(A M^{T} T\right)=A^{\prime}\left(A M^{\prime}\right)$.

So we can conclude that the matrix $H$ can be approximated by

$$
A^{\prime}=\left(\begin{array}{cccc}
\sigma_{1}\left(p^{k}\right) & 0 & \cdots & 0 \\
0 & \sigma_{2}\left(p^{k}\right) & \cdots & 0 \\
\vdots & \vdots & \ddots & \vdots \\
0 & 0 & \cdots & \sigma_{n}\left(p^{k}\right)
\end{array}\right) .
$$


In the appendix below, we have a program that gives us, for each $r \geq 3$ (each $n=2^{r-2}$, where $r \geq 3$ ), a nested lattice partition chain, which is extended by periodicity so that it is a doubly infinite chain.

This program, made by using KASH program, presents the generator and Gram matrices of the lattices in each nested lattice partition chain. We can observe that each nested lattice partition chain is extended by periodicity and such a periodicity is equal to $n=2^{r-2}$.

In this program, for each $r$ and $w$, we find the generator and gram matrices of a lattice, which is isomorphic to the canonical embedding of the ideal $\Im^{w}$, where we know that $\Im^{w}=p^{w} O_{K}$ is an ideal of $O_{K}$ generated by $p^{w}$. We can also observe that the position of this lattice compared to the $\mathbb{Z}^{n}$-lattice in the nested lattice partition chain related to $r$ is exactly $w$.

So, in this work, we construct a nested lattice partition chain related to any $r \geq 3$ (any $n=2^{r-2}$, where $r \geq 3$ ). Then, for the real case, we have the generalization to obtain a nested lattice partition chain in order to quantize the channel coefficients.

Appendix: Program for Calculating the Generator and Gram Matrices of the Lattices Associated to the Real Nested Lattice Partition Chains

The program is given as it follows:

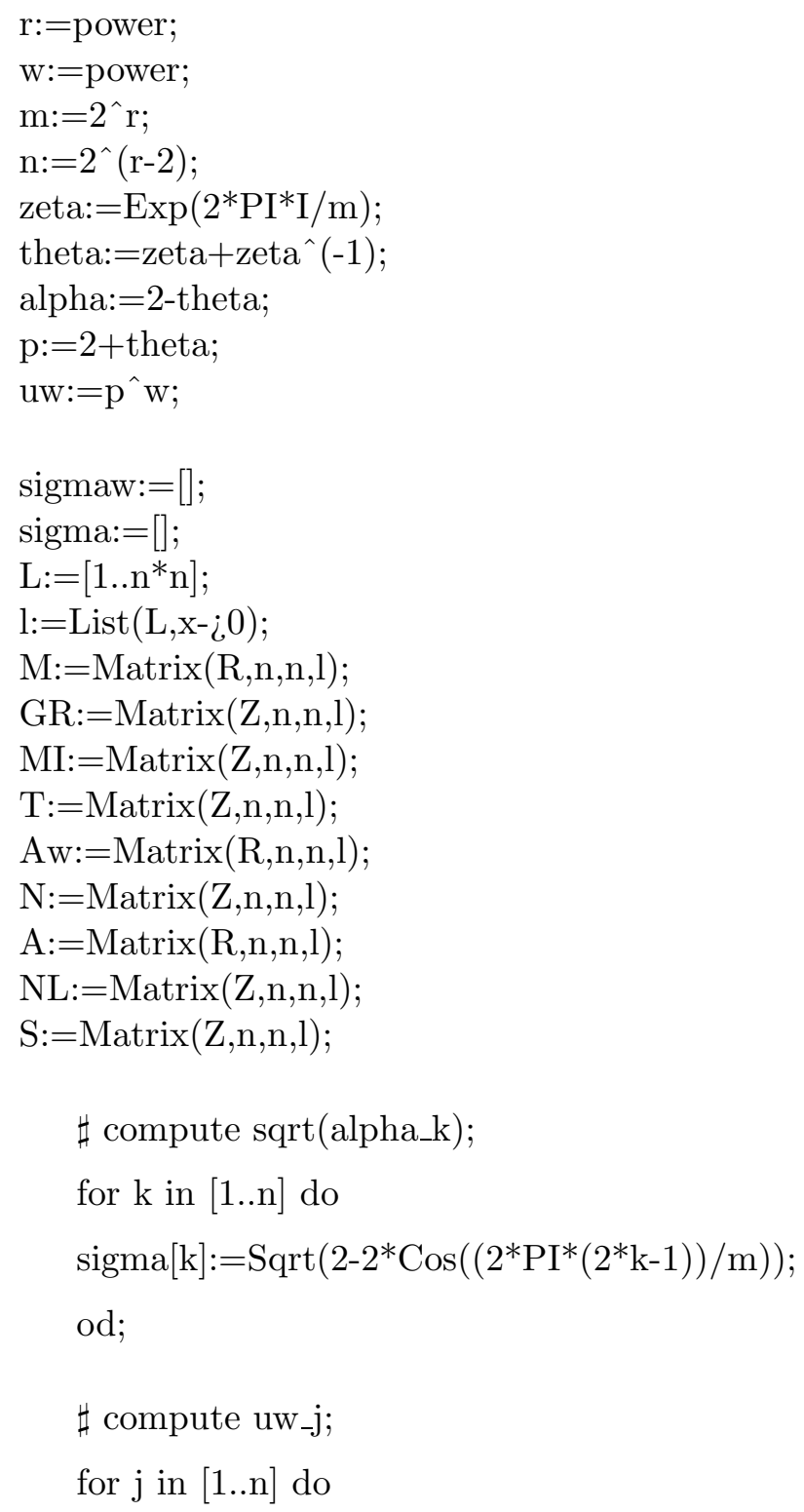


$\operatorname{sigmaw}[\mathrm{j}]:=(2+2 * \operatorname{Cos}((2 * \mathrm{PI} *(2 * \mathrm{j}-1)) / \mathrm{m}))^{\wedge} \mathrm{w}$;

od;

$\sharp$ compute A;

diag:=MatrixAlgebra(R,n);

A:=DiagonalMatrix(diag,sigma);

$\sharp$ compute Aw;

diag:=MatrixAlgebra $(\mathrm{R}, \mathrm{n})$;

Aw:=DiagonalMatrix(diag,sigmaw);

$\sharp$ compute $\mathrm{M}$;

for $\mathrm{j}$ in $[1 . . \mathrm{n}] \mathrm{do}$

$\operatorname{SetEntry}(\mathrm{M}, 1, \mathrm{j}, 1)$;

od;

for $\mathrm{i}$ in $[1 . .(\mathrm{n}-1)]$ do

for $\mathrm{j}$ in $[1 . . \mathrm{n}] \mathrm{do}$

$\operatorname{SetEntry}\left(\mathrm{M},(\mathrm{i}+1), \mathrm{j}, 2^{*} \operatorname{Cos}\left(((2 * \mathrm{PI}) / \mathrm{m}) * \mathrm{i}^{*}(2 * \mathrm{j}-1)\right)\right)$;

od;

od;

$\mathrm{MT}:=\operatorname{Transpose}(\mathrm{M})$

$\sharp$ compute $\mathrm{T}$;

for $\mathrm{j}$ in $[1 . . \mathrm{n}] \mathrm{do}$

for $\mathrm{i}$ in $[1 . .(\mathrm{n}-(\mathrm{j}-1))]$ do

$\operatorname{SetEntry}(\mathrm{T}, \mathrm{i}, \mathrm{j},-1)$;

od;

od;

$\mathrm{Mw}:=\mathrm{M} * \mathrm{~A} * \mathrm{Aw} ;$

MwT:=Transpose $(\mathrm{Mw})$;

$\mathrm{P}:=\mathrm{M}^{*} \mathrm{~A}$

Pinv: $=\mathrm{P}^{\wedge}(-1)$

$\mathrm{Mu}:=\mathrm{P}^{*} \mathrm{Aw}^{*} \mathrm{Pinv}$;

$\mathrm{MuT}:=\operatorname{Transpose}(\mathrm{Mu})$;

$\sharp$ compute MI;

for $\mathrm{i}$ in $[1 . . \mathrm{n}] \mathrm{do}$

for $\mathrm{j}$ in $[1 . . \mathrm{n}] \mathrm{do}$

$\operatorname{SetEntry}(M I, i, j, \operatorname{Round}(\mathrm{Mu}[\mathrm{i}][\mathrm{j}]]))$; 
od;

od;

MIT:=Transpose(MI);

$\mathrm{gu}:=\mathrm{MI} * \mathrm{MIT}$;

gul:=LLLGram $(\mathrm{gu})$;

$\sharp$ compute $\mathrm{N}=\mathrm{guR}$;

for $\mathrm{i}$ in $[1 . . \mathrm{n}] \mathrm{do}$

for $\mathrm{j}$ in $[1 . . \mathrm{n}] \mathrm{do}$

$\operatorname{SetEntry}(\mathrm{N}, \mathrm{i}, \mathrm{j}, \operatorname{Round}(\operatorname{gu}[\mathrm{i}][\mathrm{j}]))$;

od;

od;

$\mathrm{NL}:=\operatorname{LLLGram}(\mathrm{N})$;

$\sharp$ compute S;

for $\mathrm{i}$ in $[1 . . \mathrm{n}] \mathrm{do}$

for $\mathrm{j}$ in $[1 . . \mathrm{n}] \mathrm{do}$

$\operatorname{SetEntry}(\mathrm{S}, \mathrm{i}, \mathrm{j}, \operatorname{Round}(\operatorname{gul}[\mathrm{i}][\mathrm{j}]))$;

od;

od;

We can observe that the matrix NL is equal to the matrix S.

\section{Conclusion}

Although interference is usually viewed as an obstacle to communication in wireless networks, the authors in [2] propose a new strategy, called compute-and-forward, which exploits interference to obtain significantly higher rates between users in a network.

The idea is that compute-and-forward enables relays to decode linear equations of the transmitted messages by using the noisy linear combinations provided by the channel, that is, they do not ignore the interference as a noise. After the relays decode these linear equations, they simply send them to the destinations, which given enough equations, can recover their desired messages. The strategy is based on nested lattice codes, which are codes with a linear structure. Such structure ensures that integer combinations of codewords can be decoded reliably.

Thus, in this work, in order to realize interference alignment onto a lattice for real-valued channels, we describe a way to find a nested lattice partition chain for any dimension $n=2^{r-2}$, where $r \geq 3$.

\section{Referências}

[1] A. A. de Andrade, C. Alves and T. C. Bertoldi, Rotated Lattices via the Cyclotomic Field $\mathbb{Q}\left(\xi_{2^{r}}\right)$, in International Journal of Applied Mathematics, 3 (2006) 321-331.

[2] B. Nazer and M. Gastpar, Compute-and-forward: harnessing Interference through structured codes, in IEEE Trans. Inform. Theory, 10 (2011) 6463-6486. 